\title{
Determination of the Scattering Amplitude
}

\author{
A. D. Gangal` and J. Kupsch \\ Fachbereich Physik der Universität Kaiserslautern, D-6750 Kaiserslautern, \\ Federal Republic of Germany
}

\begin{abstract}
The problem to determine the elastic scattering amplitude from the differential cross-section by the unitarity equation is reexamined. We prove that the solution is unique and can be determined by a convergent iteration if the parameter $\lambda=\sin \mu$ of Newton and Martin is bounded by $\lambda<\lambda_{2} \simeq 0.86$. The method is based on a fixed point theorem for holomorphic mappings in a complex Banach space.
\end{abstract}

\section{Introduction}

The problem to determine the scattering amplitude when the differential crosssection is given by the integral equation of unitarity has been investigated for over 20 years [1-6] (a comprehensive list of references can be found in [7]). The unitarity equation for the elastic scattering amplitude of equal (pseudo-)scalar particles

$$
F(x)=|F(x)| e^{i \varphi(x)}=G(x) e^{i \varphi(x)}
$$

imposes a nonlinear constraint on the phase function $\varphi(x)$,

$$
G\left(\mathbf{n}_{1} \cdot \mathbf{n}_{2}\right) \cdot \sin \varphi\left(\mathbf{n}_{1} \cdot \mathbf{n}_{2}\right)=\frac{1}{4 \pi} \int d \Omega_{\mathbf{n}} G\left(\mathbf{n}_{1} \cdot \mathbf{n}\right) G\left(\mathbf{n}_{2} \cdot \mathbf{n}\right) \cos \left[\varphi\left(\mathbf{n}_{1} \cdot \mathbf{n}\right)-\varphi\left(\mathbf{n}_{2} \cdot \mathbf{n}\right)\right],
$$

where $x=\mathbf{n}_{1} \cdot \mathbf{n}_{2}=\cos \Theta \in[-1,+1]$ is the cosine of the scattering angle and $\mathbf{n}$ the unit vector in the direction of the momentum. The modulus $G(x)=|F(x)|$ is given by the differential cross-section. The characteristic quantity of this equation is the functional

$$
\lambda=\sup _{\mathbf{n}_{1}, \mathbf{n}_{2}} \frac{1}{4 \pi \mid F\left(\mathbf{n}_{1} \cdot \mathbf{n}_{2} \mid\right.} \int d \Omega_{\mathbf{n}}\left|F\left(\mathbf{n}_{1} \cdot \mathbf{n}\right)\right|\left|F\left(\mathbf{n}_{2} \cdot \mathbf{n}\right)\right| .
$$

In the following we assume that $|F(x)|$ is a continuous function without zeros. Hence the supremum (3) is a finite number. The mere existence of a continuous

^ Present address: Physics Department, Poona University, Poona, India 
solution $\varphi(x)$ of (2) has been derived under the rather restrictive condition $\lambda=\sin \mu<1$. It has been realized by Newton [3] and by Martin [4] that in this case the Schauder fixed point theorem can be applied. These proofs have been completed in $[5,6]$. The uniqueness of the solution [up to the obvious degeneracy $\varphi(x)$ and $\pi-\varphi(x)$ or $F(x)$ and $-F^{*}(x)$, respectively] has been derived by Martin [4] for $\lambda<\lambda_{1} \simeq 0.79$. This solution can be obtained by the Banach contraction mapping principle $[5,6]$ in a subspace of continuous functions of an $L^{2}$-space. With the uniform norm topology of the Banach space $C[-1,1]$ of continuous functions, the contraction mapping principle has been successfully applied only if $\lambda<\lambda_{0} \simeq 0.62($ see $[4,5])$.

For cross-sections with $\lambda>1$ the situation is more complicated. The crosssection has to satisfy additional constraints if unitary amplitudes exist. A simple constraint follows already from the optical theorem:

$$
\frac{1}{4 \pi|F(1)|} \int d \Omega_{\mathbf{n}}\left|F\left(\mathbf{n}_{1} \cdot \mathbf{n}\right)\right|^{2}<1 .
$$

So far nobody has given sufficient and necessary criteria for the existence of a solution. Concerning the uniqueness there are cross-sections with a non-trivial two-fold ambiguity of the amplitude [2] and nobody has been able to find more than two-fold ambiguities, see e.g. [8]. But a proof that no other degeneracy can occur is lacking.

In this paper the case $\lambda<1$ is reexamined. We demonstrate that the solution is unique and can be obtained by an iteration under the weaker condition $\lambda<\lambda_{2} \simeq 0.86$. The presented proof is based on a fixed point theorem for holomorphic mappings in a complex Banach space [9]. This method is interesting by itself and might be efficient also for other problems of physics where so far only the fixed point theorems of real Banach spaces have been used.

\section{The Unitarity Equation}

The elastic unitarity equation (2) can be written as

$$
\sin \varphi(x)=\int_{-1 \leqq y, z \leqq 1} d y d z H(x, y, z) \cos [\varphi(y)-\varphi(z)]
$$

with the kernel

$$
H(x, y, z)= \begin{cases}\frac{G(y) G(z)}{2 \pi G(x)} \cdot\left[1-x^{2}-y^{2}-z^{2}+2 x y z\right]_{+}^{-1 / 2} & \text { if } \quad-1<x<1, . \\ \frac{G(y) G(z)}{2 G(x)} \cdot \delta(y \mp z) & \text { if } \quad x= \pm 1 .\end{cases}
$$

For fixed $x \in(-1,1)$ the support of $H$ is the ellipse

$$
\frac{(y-z)^{2}}{2(1-x)}+\frac{(y+z)^{2}}{2(1+x)} \leqq 1,
$$

which degenerates to the straight lines $y= \pm z,-1 \leqq y, z \leqq 1$, if $x= \pm 1$. 
For the following calculations it is more convenient to use the function

$$
u(x)=\sin \varphi(x)
$$

instead of the phase function $\varphi(x)$. The unitarity equation (4) can then be written as

$$
u(x)=T[u](x),
$$

with the nonlinear mapping

$$
T[u](x)=\int d y d z H(x, y, z) \phi(u(y), u(z)),
$$

where

$$
\phi(u, v)=u v+\sqrt{1-u^{2}} \sqrt{1-v^{2}},
$$

and the positive square root is chosen for $-1 \leqq u, v \leqq 1$.

In [3-6] the fixed point equation (7) has been investigated in a Banach space of real functions $u(x)$ [or $\varphi(x)]$. But it is advantageous to extend this space to a Banach space over the complex field.

\section{The Holomorphic Unitarity Mapping}

The class of all complex valued continuous functions $x \in[-1,1] \rightarrow u(x) \in \mathbb{C}$ with the norm

$$
\|u\|=\sup _{-1 \leqq x \leqq 1}|u(x)|
$$

constitute the complex Banach space $C[-1,1]$. The ball $\{u \mid\|u\| \leqq \delta\}$ will be denoted by $\mathscr{S}_{\delta}$. If $u \in \mathscr{S}_{1}$, then $|u(x)| \leqq 1$ for all $x$ and Eq. (9) $\phi(u(y), u(z))$ is uniquely defined by analytic continuation with the square root in the half plane $\operatorname{Re} \sqrt{1-u^{2}(x)} \geqq 0$.

As kernel $H(x, y, z)$ we may choose any positive measure on the square $-1 \leqq y$, $z \leqq 1$ which depends continuously on the parameter $x \in[-1,1]$ such that

$$
h_{\psi}(x)=\int H(x, y, z) \psi(y, z) d y d z \in C[-1,1]
$$

for any continuous function

$$
\psi(y, z) \in C([-1,1] \times[-1,1]) .
$$

The characteristic quantity of the mapping (8) is the norm $\lambda$ of

$$
\left\{\begin{array}{l}
h(x)=\int H(x, y, z) d y d z \geqq 0, \\
\|h(x)\|=\lambda
\end{array}\right.
$$

which in the special case (5) coincides with (2). If $\|u\| \leqq 1$, then $|\phi(u(y), u(z))| \leqq 3$ and (8) $T[u]$ maps $\mathscr{S}_{1}$ into $\mathscr{S}_{3 \lambda}$. Moreover $T[u]$ has a bounded complex linear Fréchet derivative for any $u$ with $\|u\|<1$,

$$
\begin{aligned}
T^{\prime}[u] h= & \int d y d z(H(x, y, z)+H(x, z, y)) u(y)\left(1-\sqrt{\frac{1-u^{2}(z)}{1-u^{2}(y)}}\right) h(z), \\
& h \in C[-1,1] .
\end{aligned}
$$

It is therefore a holomorphic [10] or analytic [11] mapping of $\mathscr{S}_{\delta}, \delta<1$, into $\mathscr{S}_{3 \lambda}$. 


\section{The Fixed Point Theorem of Earle and Hamilton}

Let $\mathscr{B}$ be a Banach space with the bounded connected subsets $\mathscr{M}^{\prime} \subset \mathscr{M} \subset \mathscr{B}$. We say that $\mathscr{M}^{\prime}$ lies strictly inside $\mathscr{M}$ if there exists an $\varepsilon>0$ such that $\left\|f^{\prime}-f\right\|>\varepsilon$ for all $f^{\prime} \in \mathscr{M}^{\prime}$ and $f \in \mathscr{B} \mid \mathscr{M}$.

Theorem 1. Let $\mathscr{M}$ be a bounded connected open subset of $\mathscr{B}$.

If $T: \mathscr{M} \rightarrow \mathscr{M}$ is a holomorphic mapping and $T(\mathscr{M})$ lies strictly inside $\mathscr{M}$, then $T$ has a unique fixed point in $\mathscr{M}$ which can be obtained by iteration.

This theorem has been proved by Earle and Hamilton [9]. The norm convergence of the iteration is stated in Sect. 4 of [9]. For holomorphic mappings this theorem is more general than the contraction mapping principle.

\section{Application to the Unitarity Mapping}

Let $\mathfrak{5} \subset \mathbb{C}$ be a bounded connected domain of the complex plane. Then $\lambda \mathfrak{G}$ denotes the set $\{z=\lambda \xi \mid \xi \in \mathbb{W}\} \subset \mathbb{C}$ for real $\lambda>0$, and

$$
\mathscr{M}(\mathfrak{5})=\{f \mid f \in C[-1,1] \text { with } f(x) \in \mathfrak{G} \text { for } x \in[-1,1]\}
$$

defines a bounded connected domain of the Banach space $C[-1,1]$. If $(\mathfrak{5}$ is closed/open then $\mathscr{M}(\mathfrak{b})$ is closed/open. If $\mathfrak{G}$ is convex, then $\mathscr{M}(\mathfrak{G})$ is convex. To apply the fixed point theorem to the unitarity mapping (8) we first derive

Theorem 2. Let $\mathfrak{5}$ be a bounded connected open region of $\mathbb{C}$ and $\mathfrak{F} \subset \mathbb{C}$ be a compact convex domain which satisfies the conditions

(a) $0 \in \mathfrak{F}$ and

(b) $\phi(u, v) \in \mathfrak{F}$ if $u, v \in(\mathfrak{b}$. (8) $T$.

Then $T[u]$ maps $\mathscr{M}(\mathfrak{5})$ into $\mathscr{M}(\lambda \mathfrak{F})$, where $\lambda$ is the norm (11) of the mapping Proof. For any $u(x) \in \mathscr{M}(\mathfrak{5})$, we know that

$$
\phi(u(y), u(z)) \in \mathfrak{F} \text { for } y \in[-1,1], \quad z \in[-1,1] .
$$

The kernel $H(x, y, z)$ is positive and the domain $\mathfrak{F}$ is convex and closed. Hence the statement (b) implies that for fixed $x \in[-1,1]$ the integral

$$
\hat{u}(x)=\int H(x, y, z) \phi(u(y), u(z)) d y d z,
$$

is a complex number within the set $h(x) \cdot \mathfrak{F}$, where $h(x)$ is defined in (11). Since $0 \in \mathfrak{F}$ and $0 \leqq h(x) \leqq \lambda$, we have $h(x) \mathfrak{F} \subset \lambda \mathfrak{F}$ for $x \in[-1,+1]$, hence $\hat{u}(x) \in \lambda \mathfrak{F}$ for $x \in[-1,1]$.

The original problem to find all real fixed point solutions of $T(u)$ can be reduced to the problem to construct a bounded connected open set $(\mathfrak{G} \subset \mathbb{C}$ and a convex compact domain $\mathfrak{F} \subset \mathbb{C}$ which satisfy the conditions (a) and (b) of Theorem 2, and additionally

(c) for some $\varrho>0$, the domain $\varrho \mathfrak{F}$ lies strictly inside $(\mathfrak{5}$,

(d) the region $\mathfrak{b}$ is invariant under complex conjugation: $\left(\mathfrak{b}=\mathfrak{G}^{*}\right.$.

Lemma 1. The assumptions (a), (b), and (c) yield the existence of a unique fixed point solution of $T[u]$ within the region $\mathscr{M}(\varrho \mathfrak{F}) \subset \mathscr{M}(\mathfrak{5})$ if the norm (11) of T is bounded by $\lambda \leqq \varrho$. 
This lemma is a direct consequence of Theorem 1 and Theorem 2.

The statements (a)-(c) imply the further technical constraints:

(c') the value of $\varrho$ is restricted to $\varrho<1$,

(d) the region $(5$ encloses the real interval $[0, \varrho]$.

These constraints can be derived as follows. Since $0 \in \varrho \mathfrak{F} \subset(\mathfrak{G}$, we know from (b) that $\phi(0,0)=1 \in \mathfrak{F}$. The convexity of $\mathfrak{F}$ implies $[0,1] \subset \mathfrak{F}$, and therefore $[0, \varrho] \subset \varrho \mathfrak{F} \subset(\mathfrak{b}$. On the other hand $1 \notin \mathfrak{G}$, since $T[u]$ is no longer holomorphic if $u(x)=1$ for some $x$. The value of $\varrho$ is therefore restricted to $0<\varrho<1$ and the statements $\left(\mathrm{c}^{\prime}\right)$ and $\left(\mathrm{d}^{\prime}\right)$ are derived.

The essential consequence of all the assumptions (a)-(d) can be summarized in

Lemma 2. The unique fixed point solution of Lemma 1 is a real function and $T[u]$ has no other real continuous fixed point solutions if $\lambda \leqq \varrho<1$.

Proof. If $u(x) \in \mathscr{M}(\mathfrak{5})$ is a fixed point solution, then also the complex conjugate function $u^{*}(x)$ is a fixed point solution of $T[u]$. But (d) implies that $u^{*} \in \mathscr{M}(\mathfrak{5})$, and we know that there is only one fixed point solution within $\mathscr{M}(\mathfrak{5})$, hence $u(x)$ $=u^{*}(x)$ is real. On the other hand any real continuous fixed point solution $u(x)$ is bounded by $0 \leqq u(x) \leqq \lambda$ (see [4]). Since $[0, \lambda] \subset(5)$ if $\lambda \leqq \varrho$, see (d'), all these fixed point solutions are elements of $\mathscr{M}(\mathfrak{5})$ and Lemma 2 follows.

To complete the investigation an actual construction of sets $\mathfrak{G}$ and $\mathfrak{F}$ has to be given. Since the function (9),

$$
\xi=\phi\left(\xi_{1}, \xi_{2}\right)=\xi_{1} \xi_{2}+\sqrt{1-\xi_{1}^{2}} \sqrt{1-\xi_{2}^{2}}=\cos \left(\theta_{1}-\theta_{2}\right)
$$

if

$$
\xi_{i}=\cos \theta_{i}, \quad i=1,2,
$$

is the simple mapping

$$
\theta_{1}, \theta_{2} \rightarrow \theta= \pm\left(\theta_{1}-\theta_{2}\right)
$$

in the $\theta$-plane (the sign can be chosen such that $\operatorname{Re} \theta \geqq 0$ ) it is convenient to present such domains in the $\theta$-plane. Let $\hat{\mathfrak{F}}_{\sigma}, 0 \leqq \sigma \leqq \frac{\pi}{2}$, be the triangle

$$
\left\{\theta=\alpha+i \beta|| \beta \mid \leqq \varepsilon\left(\frac{\pi}{2}+\sigma-\alpha\right), 0 \leqq \alpha \leqq \frac{\pi}{2}+\sigma\right\},
$$

and $\hat{\mathfrak{H}}_{\sigma}$ the triangle

$$
\{\theta=\alpha+i \beta|| \beta \mid \leqq \varepsilon(\alpha-\sigma), \sigma \leqq \alpha \leqq \pi\} .
$$

The rhombus $\hat{\mathfrak{F}}_{\sigma}=\hat{\mathfrak{F}}_{\sigma} \cap \hat{\mathfrak{H}}_{\sigma}, 0 \leqq \sigma \leqq \frac{\pi}{2}$, is mapped by (15) into the triangle $\hat{\mathfrak{F}}_{0}$ :

$$
\theta_{1,2} \in \hat{\mathfrak{F}}_{\sigma} \rightarrow \theta \in \hat{\mathfrak{F}}_{0} .
$$

The images of $\hat{\mathfrak{F}}_{\sigma}$ and $\hat{\mathfrak{H}}_{\sigma}$ under the conformal mapping $\theta \rightarrow \xi=\cos \theta$ are denoted by $\mathfrak{F}_{\sigma}$ and $\mathfrak{H}_{\sigma}$, respectively. These sets obviously satisfy the relations $\mathfrak{F}_{\sigma_{1}} \subset \mathfrak{F}_{\sigma_{2}}$ and $\mathfrak{H}_{\sigma_{1}} \supset \mathfrak{H}_{\sigma_{2}}$ if $0 \leqq \sigma_{1}<\sigma_{2} \leqq \frac{\pi}{2}$. Since $\frac{\pi}{2} \in \hat{\mathfrak{F}}_{0}$ the statements (a) and (b) follow for 
$\mathbb{6}=\operatorname{int} \mathfrak{F}_{\sigma} \cap \mathfrak{H}_{\sigma}$ (open interior), $0<\sigma \leqq \frac{\pi}{2}$, and $\mathfrak{F}=\mathfrak{F}_{0}$. The convexity of $\mathfrak{F}_{0}$ is a consequence of the convexity of the boundary contour that is given by the parameter representation

$$
\xi^{ \pm}(\alpha)=x(\alpha) \pm i y(\alpha)
$$

with $x(\alpha)=\cos \alpha \cosh \varepsilon\left(\frac{\pi}{2}-\alpha\right)$ and $y(\alpha)=\sin \alpha \sinh \varepsilon\left(\frac{\pi}{2}-\alpha\right), 0 \leqq \alpha \leqq \frac{\pi}{2}$.

We have $x^{\prime}(\alpha) \leqq 0$ and

$$
x^{\prime}(\alpha) y^{\prime \prime}(\alpha)-x^{\prime \prime}(\alpha) y^{\prime}(\alpha)=\frac{1}{2}\left(1+\varepsilon^{2}\right)\left[\sinh \varepsilon\left(\frac{\pi}{2}-\alpha\right)+\varepsilon \sin 2 \alpha\right] \geqq 0,
$$

for $0 \leqq \alpha \leqq \frac{\pi}{2}$ which proves the convexity.

The statement (d) is satisfied by construction. Moreover it is obvious that $\varrho \mathfrak{F C} \mathfrak{b}$ for small values of $\varrho$. Since $\mathfrak{F}$ is compact and $\mathfrak{G}$ is open, and both sets have a smooth boundary contour of finite length, the inclusion $\varrho \mathfrak{F} \subset(\mathfrak{G}$ implies the strict inclusion needed for statement (c). The supremum $\lambda_{2}=\sup \{\lambda \mid \lambda \mathfrak{F} \subset(5)\}$ depends continuously on the parameters $\varepsilon$ and $\sigma$. It increases if $\sigma$ decreases. Unfortunately the value of $\lambda_{2}$ is not given by the extremal points on the real axis; it has to be calculated numerically. We have evaluated the limit case $\sigma=0$. The problem is then to determine

$$
\lambda_{2}=\sup \left\{\lambda \mid \lambda \mathfrak{F}_{0} \subset \mathfrak{H}_{0}\right\}=\sup \left(\frac{\cos \alpha_{1} \cdot \cosh \varepsilon \alpha_{1}}{\cos \alpha_{2} \cdot \cosh \varepsilon\left(\frac{\pi}{2}-\alpha_{2}\right)}\right)
$$

under the subsidiary condition

$$
\tan \alpha_{1} \cdot \tanh \varepsilon \alpha_{1}=\tan \alpha_{2} \cdot \tanh \varepsilon\left(\frac{\pi}{2}-\alpha_{2}\right) \text { for } 0 \leqq \alpha_{1}, \alpha_{2} \leqq \frac{\pi}{4} .
$$

The numerical result is $\lambda_{2}=0.863934$ within an accuracy of $10^{-6}$ for all $\varepsilon \leqq 0.001$.

It is of course possible to choose the domain $(\mathfrak{5}$ and henceforth $\mathfrak{F}$ in a different way. We have tried several alternatives, but we did not succeed to construct sets $\mathbb{6}$ and $\mathfrak{F}$ with a larger value of $\lambda_{2}$.

\section{References}

1. Puzikov, L., Ryndin, R., Smorodinskii, Ia.: Reconstruction of the scattering matrix of a twonucleon system. Sov. Phys. JETP 5, 489 (1957)

Kopylov, G.I., Lomakina, Z.D.: On the problem of the direct reconstruction of the elasticscattering amplitude. Sov. Phys. JETP 11, 1188 (1960)

Klepikov, N.P.: On the completeness of the "complete scattering experiment". Sov. Phys. JETP 20, 505 (1964)

2. Crichton, J.H.: Phase-shift ambiguities for spin-independent scattering. Nuovo Cimento 45A, 256 (1966) 
3. Newton, R.G.: Determination of the amplitude from the differential cross section by unitarity. J. Math. Phys. 9, 2050 (1968)

4. Martin, A.: Construction of the scattering amplitude from the differential cross-sections. Nuovo Cimento 59A, 131 (1969)

Martin, A.: Scattering theory: Unitarity, analyticity and crossing. In: Lecture Notes in Physics, Vol. 3. Berlin, Heidelberg, New York: Springer 1969

5. Atkinson, D., Johnson, P.W., Warnock, R.L.: Determination of the scattering amplitude from the differential cross-section and unitarity. Commun. Math. Phys. 28, 133 (1972)

6. Tortorella, M.: Existence and uniqueness questions for the unitarity equation. J. Math. Phys. 13, 1764 (1972); The unitarity equation for scattering in the absence of spherical symmetry. 15, 745 and Addendum 1153 (1974); Experimental uncertainties in the problem of the unitarity equation 16, 823 (1975)

7. Newton, R.G.: Scattering theory of waves and particles, 2nd edn. Berlin, Heidelberg, New York: Springer 1982

8. Itzykson, C., Martin, A.: Phase-shift ambiguities for analytic amplitudes. Nuovo Cimento 17A, 245 (1973)

Cornille, H., Drouffe, J.M.: Phase-shift ambiguities for spinless and $L_{\max } \leqq 4$ elastic scattering. Nuovo Cimento 20A, 401 (1974)

9. Earle, C.J., Hamilton, R.S.:Proc. Symp.Pure Math.16,61, Providence: Am. Math. Soc.(1968)

10. Hille, E., Phillips, R.S.: Functional analysis and semigroups. Providence: Am. Math. Soc. 1957

11. Berger, M.S.: Nonlinearity and functional analysis. New York, San Francisco, London: Academic Press 1977

Communicated by A. Jaffe

Received October 5, 1983; in revised form December 14, 1983 
